\title{
Marsupials that model melanoma
}

\author{
by Monica Harrington
}

SCIENTIFIC NAME

Monodelphis domestica

TAXONOMY

PHYLUM: Chordata

CLASS: Mammalia

ORDER: Didelphimorphia

FAMILY: Didelphidae

\section{Physical description}

The gray short-tailed opossum (Monodelphis domestica) is a small marsupial native to South America. It inhabits forests, dry scrubland and agricultural environments in the eastern central part of the continent, south of the Amazon River. In the wild, individuals typically grow to $12-18 \mathrm{~cm}$ in length and weigh $80-100 \mathrm{~g}$, but those kept in captivity may become much larger. As suggested by the common name, their tails are proportionately shorter than in some opossums, measuring about half the length of the body, and most of their fur is grayish brown.

Opossums have well-developed, curved claws and sharp teeth. They are omnivorous, hunting invertebrates, amphibians and reptiles as well as eating fruit and scavenging dead animals. A nocturnal species, opossums are most active during the first few hours after dusk. Opossums are typically solitary.

\section{Reproduction}

Opossums can breed throughout the year and produce as many as six litters, each consisting of 6-13 pups. The gestation period is about 14 days, and the pups are not fully developed when born, as with other marsupials. Unlike other marsupials, however, $M$. domestica females do not have pouches. Instead, the offspring, which are only about $1 \mathrm{~cm}$ in length and $0.1 \mathrm{~g}$ in weight, attach themselves directly to the female's teats for several weeks.

\section{Research résumé}

Several characteristics of the gray short-tailed opossum make it an amenable research model. It is small and docile; can be maintained and bred relatively easily in laboratory settings ${ }^{1,2}$; and produces large litters of neonates that are readily accessible, owing to the absence of a pouch in the female. $M$. domestica can be considered a 'prototype'

species for basic research on marsupial biology, much the same as Mus musculus, the laboratory mouse, is a prototype for basic research on the biology of placental mammals ${ }^{3}$. In part because of this status, $M$. domestica was the first marsupial to have its genome sequenced ${ }^{4}$.

Because the neonatal immune system is not fully developed, as in other marsupials, $M$. domestica pups are useful models for transplantation studies and cancer research ${ }^{5,6} . M$. domestica is the only mammal, other than humans, that is susceptible to melanoma in response to ultraviolet radiation alone ${ }^{3}$.

$M$. domestica is also used as a model for diet-induced hypercholesterolemia and hyperlipidemia ${ }^{3,7}$. There is extensive variation in response to dietary fat and cholesterol among individual opossums, which seems to be largely attributable to a single gene? Clarifying the role of this gene may lead to a better understanding of dietary lipoprotein physiology in humans. Furthermore, the opossum's omnivorous diet more closely resembles a typical human diet and may therefore make $M$. domestica a better model than conventional laboratory animals for research on physiological effects of dietary fat and cholesterol ${ }^{3}$.

Finally, newborn opossum pups have a unusual ability to recover from severe spinal cord injuries and have been used to study regeneration of the nervous system ${ }^{8,9}$.

1. Allison, S.O., Criley, J.M., Kim, J.Y. \& Goodly, L.J. Cage change intervals for opossums (Monodelphis domestica) in individually ventilated cages. J. Am. Assoc. Lab. Anim. Sci. 50, 647-652 (2011).

2. Rousmaniere, H. Husbandry of Monodelphis domestica in the study of mammalian embryogenesis. Lab Anim. (NY) 39, 219-226 (2010).

3. VandeBerg, J.L. \& Robinson, E.S. The laboratory opossum (Monodelphis domestica) in laboratory research. ILAR J. 38, 4-12 (1997).

4. Mikkelsen, T.S. et al. Genome of the marsupial Monodelphis domestica reveals innovation in non-coding sequences. Nature 447, 167-177 (2007).

5. Wang, Z., Hubbard, G.B., Pathak, S. \& VandeBerg, J.L. In vivo opossum xenograft model for cancer research. Cancer Res. 63, 6121-6124 (2003).

6. Wang, Z., Hubbard, G.B., Clubb, F.J. \& VandeBerg, J.L. The laboratory opossum (Monodelphis domestica) as a natural mammalian model for human cancer research. Int. J. Clin. Exp. Pathol. 2, 286-299 (2009).

7. Kushwaha, R.S., VandeBerg, J.F. \& VandeBerg, J.L. Effect of dietary cholesterol with or without saturated fat on plasma lipoprotein cholesterol levels in the laboratory opossum (Monodelphis domestica) model for dietinduced hyperlipidaemia. Br. J. Nutr. 92, 63-70 (2004).

8. Wheaton, B.J., Noor, N.M., Dziegielewska, K.M., Whish, S. \& Saunders, N.R. Arrested development of the dorsal column following neonatal spinal cord injury in the opossum, Monodelphis domestica. Cell Tissue Res. doi:10.1007/ s00441-014-2067-6 (published online 9 December 2014).

9. Saunders, N.R. et al. Age-dependent transcriptome and proteome following transection of neonatal spinal cord of Monodelphis domestica (South American grey short-tailed opossum). PLoS ONE 9, e99080 (2014). 